Article

\title{
Structural Characterization by NMR of a Double Phosphorylated Chimeric Peptide Vaccine for Treatment of Alzheimer's Disease
}

\author{
Karla Ramírez-Gualito ${ }^{1, *}$, Monique Richter ${ }^{2}$, Manolis Matzapetakis ${ }^{3}$, David Singer ${ }^{2}$ and \\ Stefan Berger ${ }^{1}$
}

1 Institute of Analytical Chemistry, University Leipzig, Johannisallee 29, Leipzig 04103, Germany; E-Mail: stberger@rz.uni-leipzig.de

2 Institute of Bioanalytical Chemistry, University Leipzig, Deutscher Platz 5, Leipzig 04103, Germany; E-Mails: monique.richter@bbz.uni-leipzig.de (M.R.); david.singer@arcor.de (D.S.)

3 Instituto de Tecnologia Química e Biológica, Universidade Nova de Lisboa. Av. da República, Oeiras 2780-157, Portugal; E-Mail: matzman@itqb.unl.pt

* Author to whom correspondence should be addressed; E-Mail: karamirezg@prodigy.net.mx; Tel.: +49-341-973-6101; Fax: +49-341-971-1833.

Received: 7 February 2013; in revised form: 19 April 2013 / Accepted: 22 April 2013 /

Published: 26 April 2013

\begin{abstract}
Rational design of peptide vaccines becomes important for the treatment of some diseases such as Alzheimer's disease (AD) and related disorders. In this study, as part of a larger effort to explore correlations of structure and activity, we attempt to characterize the doubly phosphorylated chimeric peptide vaccine targeting a hyperphosphorylated epitope of the Tau protein. The 28-mer linear chimeric peptide consists of the double phosphorylated B cell epitope Tau $229-237[\mathrm{pThr} 231 / \mathrm{pSer} 235]$ and the immunomodulatory $\mathrm{T}$ cell epitope $\mathrm{Ag} 85 \mathrm{~B}_{241-255}$ originating from the well-known antigen Ag85B of the Mycobacterium tuberculosis, linked by a four amino acid sequence -GPSL-. NMR chemical shift analysis of our construct demonstrated that the synthesized peptide is essentially unfolded with a tendency to form a $\beta$-turn due to the linker. In conclusion, the -GPSL- unit presumably connects the two parts of the vaccine without transferring any structural information from one part to the other. Therefore, the double phosphorylated epitope of the Tau peptide is flexible and accessible.
\end{abstract}


Keywords: NMR spectroscopy; Biological Magnetic Resonance Data Bank; Alzheimer's disease; peptide vaccine; B cell epitope; T cell epitope; Tau protein; hyperphosphorylation; Mycobacterium tuberculosis

\section{Introduction}

The conformation of a peptide or protein can be relevant to its stability and function. Various intramolecular interactions are important for the relationship between the primary and tertiary structure. Structure determination of small peptides is a challenging task but necessary to obtain insights into their ability to bind to certain receptors; e.g., major histocompatibility complex (MHC) involved in immune response. To understand the relation between structure and function of peptides, it is necessary to consider folding initiation sites such as $\beta$-hairpin motifs [1] as well as $\alpha$-helical and $\beta$-sheet like structures. It should also be taken into account that medium-range peptides commonly exist in solution as complex mixtures of conformers of similar energies and correlation times. This inherent inhomogeneity often renders them difficult to study and determine specific interactions through the space associated with certain tertiary structural elements [2]. Nevertheless, recent methodologies are able to uncover evidence of small populations of folded structures in such seemingly unfolded ensembles in water [3,4]. Intrinsically disordered proteins (IDPs) have been shown to be functional, despite their lack of well-defined structure, imposing a new perspective on the relationship between primary protein sequence and function and necessitating the development of an entirely new set of experimental and analytical techniques $[5,6]$. Analysis of chemical shifts, by identifying the deviations of the chemical shifts of certain nuclei from random coil value, is one useful tool for detecting secondary structure elements and has been commonly used in the characterization of proteins by NMR [7-10]. With this, NMR analysis becomes a useful tool for the study of local conformational preferences that encode biological functions [11-13].

$\beta$-turns are the most common type of non-repetitive structures recognized in proteins and are important for providing a directional change within the polypeptide chain [14]. There is also much evidence that $\beta$-turns are the key structures for molecular recognition and protein folding [15]. Since they were first recognized, much effort has been made for their analysis and for prediction of their presence from a specific amino acid sequence [16].

The rational design of peptide based vaccines has become an important target for the treatment of infectious diseases, cancer and neurodegenerative diseases like the Alzheimer's disease (AD) [17-19]. $\mathrm{AD}$ is the most common form of dementia that is placing a considerable and increasing burden on patients, caregivers and the society. AD is clinically characterized by a progression from episodic memory problems to a global decline of cognitive function including the inability to acquire or recall memories, motor dysfunctions and personality changes [20]. The impairment of cognitive function in patients suffering from $\mathrm{AD}$ is accompanied by two pathological protein aggregates found in their brain. These aggregates are commonly referred as senile plaques (SP) and neurofibrillary tangles (NFT's). SP are extracellular deposits made up by the $\beta$-amyloid peptide whereas NFT's mainly consist of hyperphosphorylated Tau protein, which aggregates within neurons [21]. The biochemical cause of the 
disease still remains to be understood but there is an agreement that reducing the plaque and tangle burden e.g., by immunotherapy, is beneficial for the patients [22,23]. Due to severe difficulties in AD related immunization trials, alternatives, such as the rational design of anti-A $\beta$ and anti-phospho Tau peptide vaccines, have become very important [24,25].

The 28-mer chimeric AD-specific peptide vaccine reported in this study is composed of the doubly phosphorylated Tau B cell epitope $\mathrm{Tau}_{229-237}[\mathrm{pThr231/pSer235]}$ and an immunomodulatory $\mathrm{T}$ cell epitope originating from the antigen Ag85B of the Mycobacterium tuberculosis. In our construct, both epitopes have been linearly connected via a four amino acid linker, which was previously utilized for the design of such short peptide vaccines [26-28]. The aim of the present study was to associate a potential correlation between the immunological properties of the vaccine and its conformation. We report the measurement and analysis of the NMR chemical shifts of ${ }^{1} \mathrm{H},{ }^{13} \mathrm{C}$ and ${ }^{15} \mathrm{~N}$ to study the conformational state of the 28 -mer peptide vaccine and its artificial loop.

\section{Results and Discussion}

The peptide vaccine (Figure 1) comprised of 28 amino acids can be divided in three sections, the immunomodulatory $\mathrm{T}$ cell epitope $\mathrm{Ag} 85 \mathrm{~B}_{241-255}$ from Mycobacterium tuberculosis, the four amino acid linker sequence -GPSL-, and the B cell epitope $\mathrm{Tau}_{229-237}[\mathrm{pThr} 231 / \mathrm{pSer} 235]$. The double phosphorylated Tau epitope is thought to be AD specific and a promising target for immunotherapy [24,29]. The peptide was synthesized by standard Fmoc-chemistry and was post-synthetically phosphorylated using phosphoramidite. The purity was above 95\% confirmed by analytical HPLC and MALDI mass spectrometry (Figure 1).

The spectra collected were analyzed using the CCPNmr software [30] and we were able to complete $85.7 \%$ of the type-specific assignment of 24 out of the 28 spins systems involved. Gln1, Asp2, Pro27 and Ser28, could not be observed, likely due to increased flexibility and exchange with the bulk solvent in these parts of the molecule that rendered the specific resonances of these residues invisible to NMR. The assignment for $85.0 \%$ of the hydrogen atoms, $66.9 \%$ of the carbon atoms and $64.9 \%$ of the nitrogen atoms was made. Hydrogen assignment corresponds to $64.2 \%$ assigned hydrogen atoms of the backbone and $83.9 \%$ of the side chain. Analysis of the NOESY spectrum revealed only short range NOEs between neighboring residues $(i, i+1$ and $i, i+2)$ but no long range ones. 35 NOEs of various types of $(i, i+1)$ and 7 of $(i, i+2)$ ranges were identified. More specifically $d \gamma N(i, i+2)$ were identified between Asp15 and Val13, d $\gamma \alpha(\mathrm{i}, \mathrm{i}+2)$ from Val20 to Thr22, $\mathrm{d} \delta \mathrm{N}(\mathrm{i}, \mathrm{i}+2)$ between $\operatorname{Arg} 21$ and Leu19, d $\beta \mathrm{N}(\mathrm{i}, \mathrm{i}+2)$ from Arg21 to Pro23, d $\gamma \mathrm{N}(\mathrm{i}, \mathrm{i}+2)$ from Thr22 to Val20, d $\alpha \beta(\mathrm{i}, \mathrm{i}+2)$ between Pro23 and Arg21 and finally $\mathrm{d} \beta \mathrm{N}(\mathrm{i}, \mathrm{i}+2)$ from Lys 25 to Pro23. These NOEs are found in the regions bracketing the Gly16, Pro17, Ser18 and Leu19, GPSL hypothesized linker suggesting a specific spatial order of the peptide. However, the absence of longer range NOEs implies an overall conformational inhomogeneity of the construct. We assume that the artificial linker (Figure 2) is forcing both arms of the peptide chain into a more limited conformational space while not significantly affecting the overall flexibility of the active epitopes, as is also evident from the very small chemical shift deviations from the random coil of those positions [31]. Efforts to study the conformational exchange by variations of temperature were limited by the $\mathrm{H}_{2} \mathrm{O} / \mathrm{D}_{2} \mathrm{O}$ working temperature range and by the solubility of the peptide so it was not possible to reach conclusions by temperature dependent 
NMR studies. In addition, the possibility of our observations originating from spin diffusion is not significant since the mixing times used for NOESY (Nuclear Overhauser Effect SpectroscopY) are too small to cause spin diffusion.

Figure 1. Amino acid sequence of the AD-specific peptide vaccine ${\mathrm{Ag} 85 \mathrm{~B}_{241-255} \text {-GPSL-Tau } 229-237}[\mathrm{pThr231/pSer235]}$ (upper left panel). The Tau sequence is numbered according to the longest human isoform with 441 amino acids. For convenience the peptide was renumbered from 1 to 28 from N- to C-terminus. RP-HPLC chromatogram (upper right panel) and MALDI mass spectrum (lower panel) of the purified

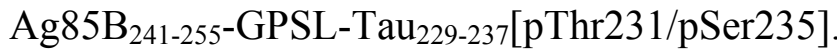
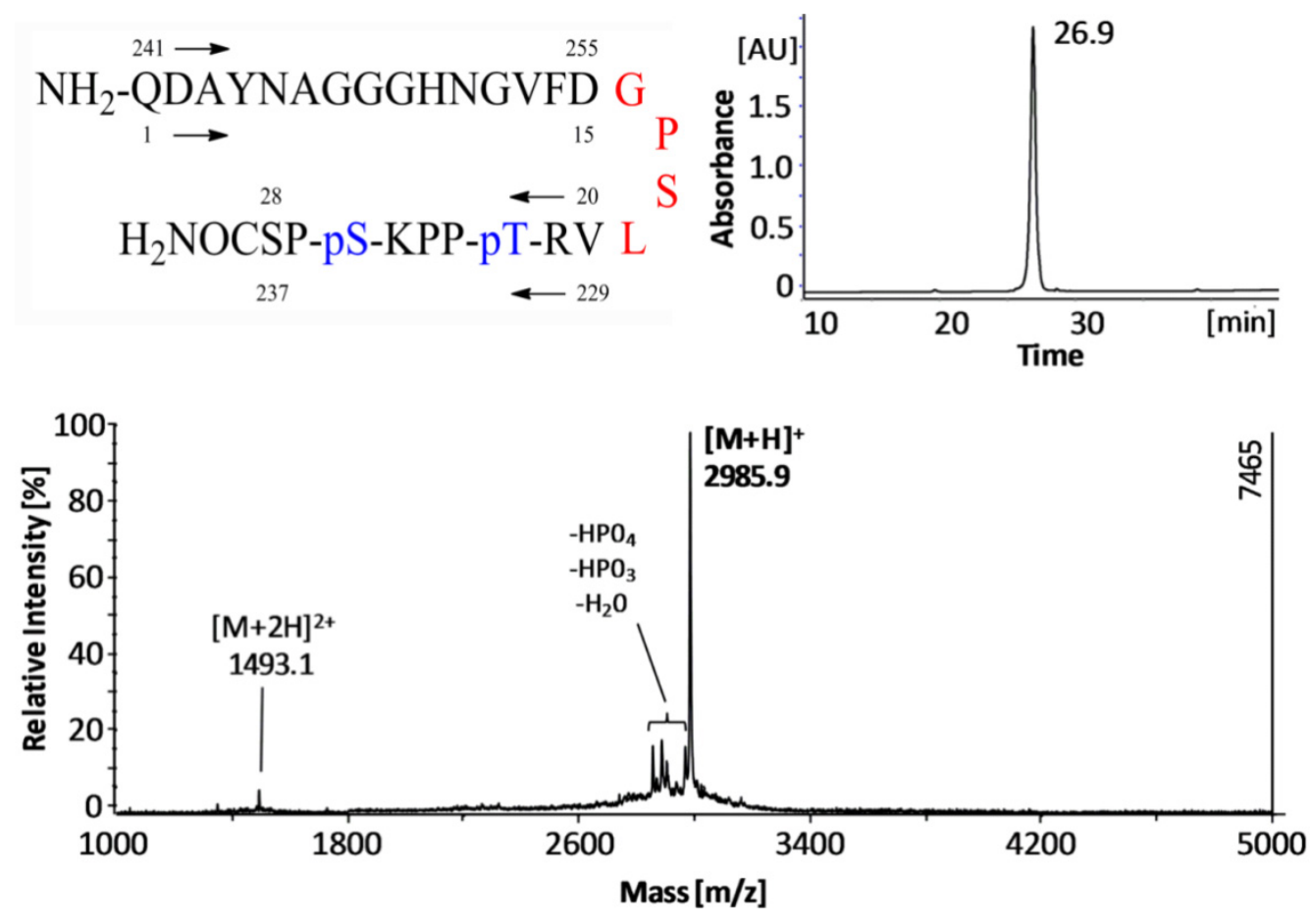

Figure 2. Schematic diagram of the loop formed by the residues Gly16-Leu18.

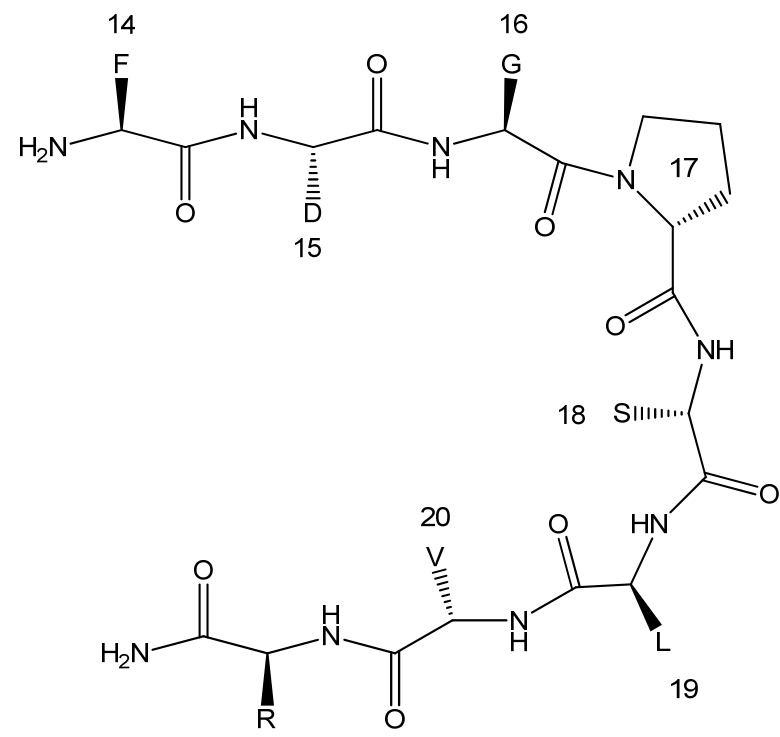


The GPSL linker, has been previously used to stabilize other peptidic structures, e.g., $\alpha$-helical structures of such short peptide vaccines. Additionally, it was used as a flexible connection for peptides, which adopt their tertiary structure independently [26-28]. In the loop the residues glycine and proline potentiate a $\beta$-turn in the polypeptide, whereas the side-chain of serine will favor hydrogen bonds with the free $\mathrm{H}^{\mathrm{N}}$ of the backbone. The side chain of leucine in the sequence is important for hydrophobic interactions [26].

Due to the lack of long range NOEs in our system and in order to better probe its structural features, we attempted to also compare the chemical shift differences of several atoms of the amino acids of the peptide sample with random coil chemical shifts, to identify any significant deviations statistically. The chemical shifts of our system are reported in the Supplementary Information for the ${ }^{1} \mathrm{H},{ }^{13} \mathrm{C}$ and ${ }^{15} \mathrm{~N}$ atoms (Table S1) and are deposited in BMRB (BMRB ID = 19112) [32]. In the ${ }^{15} \mathrm{~N}-\mathrm{HSQC}$ (Heteronuclear Single Quantum Coherence) spectrum (Figure 3) a group of four glycines (Gly6, Gly8, Gly9 and Gly12) was identified, the $\mathrm{H}^{\mathrm{N}}$ had ${ }^{1} \mathrm{H}$ and ${ }^{15} \mathrm{~N}$ chemical shifts between 8.2 to 8.5 ppm and between 107 to $109 \mathrm{ppm}$, respectively. In contrast, the $\mathrm{H}^{\mathrm{N}}$ of Gly16 had a ${ }^{1} \mathrm{H}$ chemical shift of $7.8 \mathrm{ppm}$ suggesting this amino acid experienced a different environment compared to the remaining glycines in the peptide, implying a potential function within the GPSL loop. We excluded the possibility of aggregation being a factor since we did not observe any chemical shift dependence in measurements made on samples of concentrations different by an order of magnitude. In addition, diffusion measurements did not suggest aggregation.

Figure 3. ${ }^{15} \mathrm{~N}-\mathrm{HSQC}$ spectrum of the AD-specific peptide vaccine (Ag85B $241-255-G P S L-T a u$ 229-237[pThr231/pSer235]).

${ }^{15} \mathrm{~N}-\mathrm{HSQC}$ spectrum

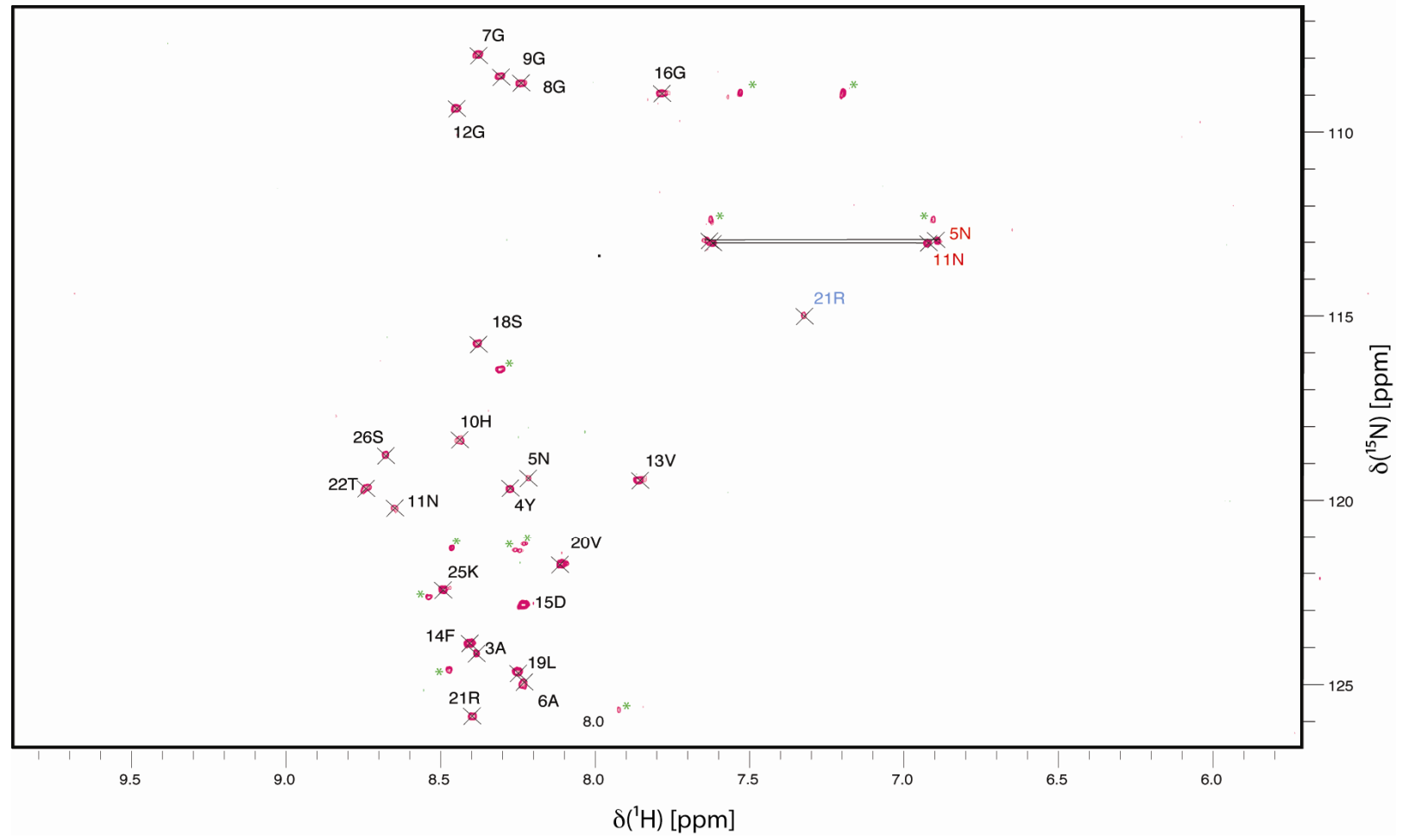

The labels in red correspond to the side chain amide of Asn5 and Asn11 while the peak in blue to the aliased peak of Arg21 whose ${ }^{15} \mathrm{~N}$ resonance is normally at $84.99 \mathrm{ppm}$. The peaks marked with a green asterisk could not be assigned and were not part of the main chain. 
In addition to the above example of the Gly16, a wider statistical analysis was performed in order to quantify the chemical shift information of the complete amino acid sequence. The protons most sensitive to conformational changes associated with secondary structure are the $\mathrm{H} \alpha$, which have been widely used to study the conformation of peptides and for the determination of structural elements in proteins and peptides folding studies [26,33-35]. In addition, the chemical shifts of $\mathrm{C} \alpha$ and $\mathrm{C} \beta$ have a significant correlation with dihedral angles and are often used for the structural analysis of peptides and proteins [36]. Moreover $\mathrm{H}^{\mathrm{N}}$ have been used as probes of a consistent pattern for $\beta$-hairpins and three-stranded $\beta$ sheets [7]. Since the number of identified or available chemical shifts for each amino acid was not the same, we opted to use a combined analysis of the differences with the use of the RMSS (Root of the Mean Sum of the Squares) value. This analysis yields a single value for each amino acid representing its cumulative deviation from random coil behavior and due to the use of weighted averages it is independent of the number of chemical shifts it is composed of. The results of the statistical analysis (Figure 4A) showed that some populations deviate from the random coil values in agreement with the presence of a partial secondary structure present in the 28 -mer peptide. The most important changes were observed in the region from Phe14 to Val20. Also, the chemical shifts revealed a significant difference in the region comprising the GPSL sequence. Another important feature was observed for pThr22 and pSer26, the two phosphorylated amino acids, which have chemical shifts that differ from the non-phosphorylated ones. Interestingly, amino acids Phe14 and Asp15 showed a difference from the random coil chemical shifts, but this may be attributed to the ring current effect of the Phe aromatic ring over Asp15.

Figure 4. (A) Graphical representation of the chemical shift differences versus random coil chemical shifts of the 28-mer peptide calculated through the RMSS. (B) Chemical shift differences for $\mathrm{H}^{\mathrm{N}}, \mathrm{H} \alpha$ and $\mathrm{H} \beta$. (C) Chemical shift differences for $\mathrm{CO}, \mathrm{C} \alpha$ and $\mathrm{C} \beta$. B and $\mathbf{C}$ were calculated considering only the artificial loop region chemical shifts and random coil values.
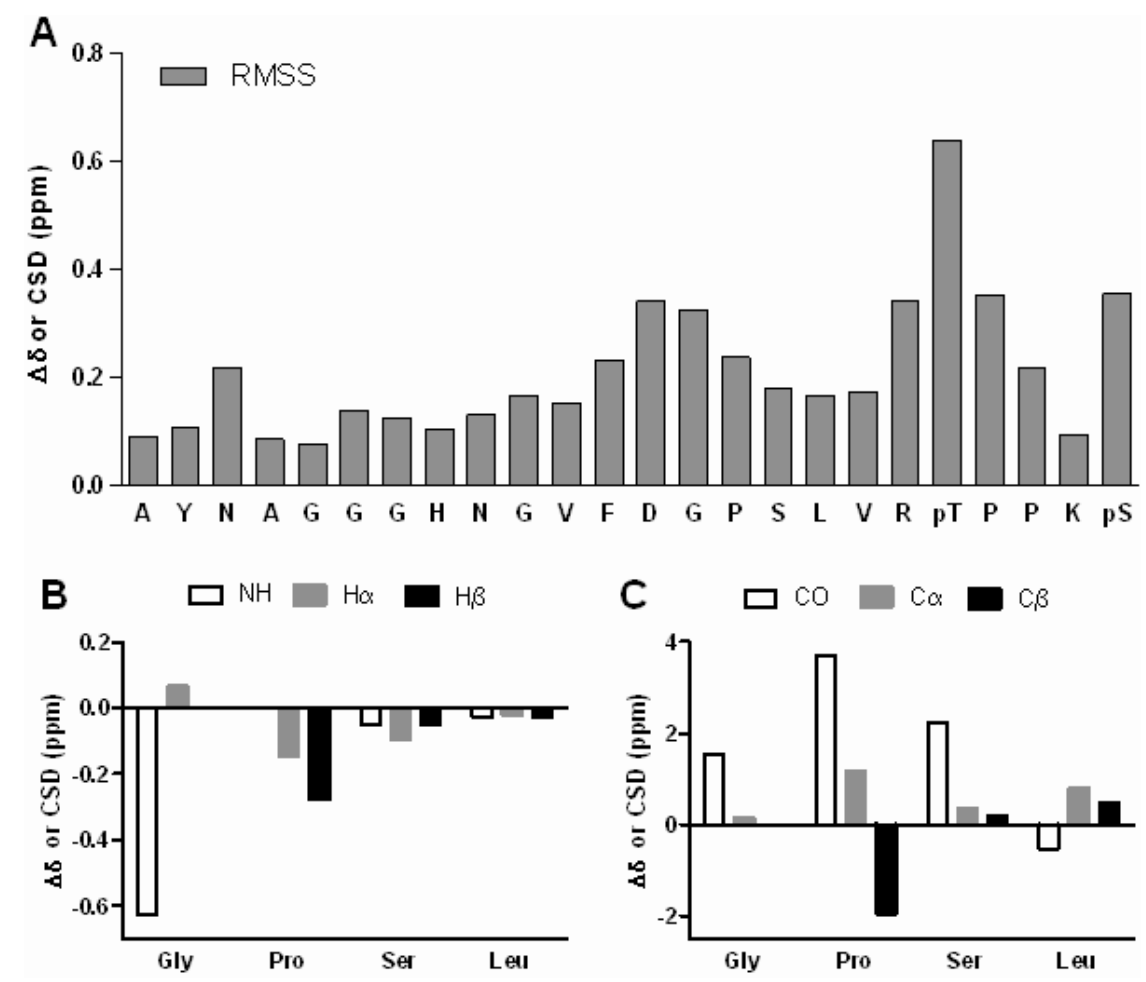
A specific analysis was done using the chemical shift difference of the loop sequence GPSL. The chemical shift difference $(\Delta \delta)$ was defined as $\Delta \delta=\delta_{\text {exp }}-\delta_{\text {rc }}$, where $\delta_{\text {exp }}$ belongs to the experimental chemical shift and $\delta_{\text {rc }}$ to the random coil shift. Several studies support the argument that $\Delta \delta$ could be indicative for the identification of $\alpha$-helices, $\beta$-sheets and loops, but such an analysis depends on the definition of the random coil state for a given amino acid sequence which is not easy to reach $[35,37,38]$. The random coil state has been defined as the state where the dihedral angles of each residue is independent of the conformation of the neighboring residues, but some effects such as solvation and ring current effects will often influence local chemical shifts [39]. The results for the chemical shift differences for the atoms $\mathrm{H}^{\mathrm{N}}, \mathrm{H} \alpha$ and $\mathrm{H} \beta$ (Figure 4B) are consistent with the corresponding results for chemical shift analysis. As previously mentioned, the most significant difference was observed for the $\mathrm{H}^{\mathrm{N}}$ atom of Gly16, supporting its special role in the GPSL loop. In the case of the C atoms (Figure 4C), the results are also consistent displaying significant differences for Gly16, Pro17 and Ser18. Only for Leu19 it was not possible to observe any significant shift differences for all protons and $\mathrm{C}$ atoms.

${ }^{3} J_{\mathrm{HN}-\mathrm{H} \alpha}$ coupling constant carries structural information and has a long recognized relation with the $\varphi$ dihedral angle of the peptide plane. That relation is described by the parameterized Karplus relationship [40]. We were able to extract these values from the analysis of the DQF-COSY (Double Quantum Filter-COrrelationSpectroscopY). However, the evaluation of these Js turned to be difficult in the absence of an estimate of the expected values for the random coil of our peptide. For this purpose we used, the Flexible-Meccano program to calculate 100,000 random structures of our designed peptide that were not in violation of the Ramachandran space and subsequently calculate the average Js over all of the calculated structures using the Karplus relationship. We were then able to compare these values with those extracted from the COSY and the results of the differences between these two sets of values in shown in Figure 5 (Table S2). Only negligible difference between the experimental and the theoretical values can be observed suggesting that the ${ }^{3} J_{\mathrm{HN}-\mathrm{H \alpha}}$ couplings are less sensitive than chemical shift for the detection of structural features.

Figure 5. Graphical representation of the scalar coupling differences $(\Delta J)$ of the experimental data from those calculated by Flexible-Meccano $\left(\Delta J=J_{\mathrm{Exp}}-J_{\mathrm{FM}}\right)$.

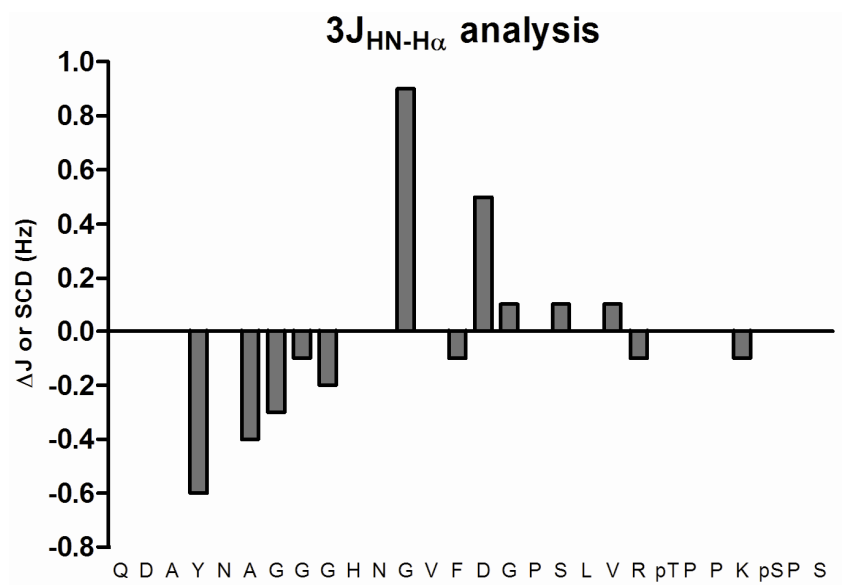




\section{Experimental}

\subsection{Peptide Synthesis}

The phosphorylated Ag85B241-255-GPSL-Tau229-237[pThr231/pSer235] peptide was synthesized by standard Fmoc/t-butyl chemistry using a polystyrene-based Rink Amide (MBHA) resin $(0.65 \mathrm{mmol} / \mathrm{g}$, MultiSynTech GmbH, Witten, Germany). Fmoc-protected amino acids (0.5 mol/L, MultiSynTec $\mathrm{GmbH}$ ) were activated with diisopropylcarbodiimide in the presence of 1-hydroxybenzotriazole (DIC/HOBt) and coupled automatically in eight molar excess by the Syro2000 multiple peptide synthesizer (MultiSynTech $\mathrm{GmbH}$ ). Amino acids to be phosphorylated were incorporated with unprotected side chains, i.e., Fmoc-Ser-OH and Fmoc-Thr-OH. Global postsynthetic phosphorylation was done as described elsewhere [23]. In brief, free hydroxyl groups were phosphitylated with 10 equivalents of dibenzyl- $N, N$-diisopropyl-phosphoramidite in the presence of 20 equivalents of $1 \mathrm{H}$-tetrazole in acetonitrile/dimethylformamide at room temperature for $90 \mathrm{~min}$ and $16 \mathrm{~h}$. After washing, phosphitylated amino acids were oxidized with 100 eq tert-butyl hydroperoxide $(t-\mathrm{BuOOH})$ in decane at room temperature for $90 \mathrm{~min}$. After synthesis, the peptidyl resin was washed with dimethylformamide and methylene chloride, air dried and the peptide was cleaved with 5\% water, $4 \%$ thioanisol, $4 \% \mathrm{~m}$-cresol, and $2 \%$ ethanedithiol in trifluoroacetic acid (TFA) at room temperature for 2 h. Peptides were precipitated with ice cold diethyl ether, washed three times, and air dried. The peptide was purified on an Äkta HPLC System (Amersham Bioscience GmbH, Freiburg, Germany) using a Jupiter C18-column (21.2 $\mathrm{mm} \times 250 \mathrm{~mm}$, Phenomenex Inc., Torrance, CA, USA). Elution was performed by a linear gradient with an increase of $1 \%$ acetonitrile per minute in the presence of $0.1 \%$ TFA $(10 \mathrm{~mL} / \mathrm{min}, 220 \mathrm{~nm})$. The purity of the peptide was confirmed by analytical RP-HPLC using a Jupiter C18 column $(2.0 \times 150 \mathrm{~mm}, 3 \mu \mathrm{m}, 300 \AA)$ and the correct masses confirmed by matrix-assisted laser desorption/ionization time-of-flight mass spectrometry (MALDI-TOF-MS; 4700 proteomic analyzer; Applied Biosystems GmbH, Darmstadt, Germany) operated in positive ion-mode using $\alpha$-cyano-4-hydroxy-cinnamic acid (BrukerDaltonics GmbH, Bremen, Germany). Alternatively, peptide purity was confirmed by LC-ESI-MS (Qstar pulsar, ESI QqTOF Hybrid mass spectrometer, Applied Biosystems) using acetonitrile/water as eluents in the presence of $0.1 \%$ formic acid. The peptide was obtained in a yield of $14 \%$.

\subsection{NMR Spectroscopy}

The NMR sample were prepared by dissolving the peptide in $500 \mu \mathrm{L}$ phosphate buffer $\left(\mathrm{Na}_{2} \mathrm{HPO}_{4} / \mathrm{NaH}_{2} \mathrm{PO}_{4}\right)$ at $\mathrm{pH} 5.8$ with a 9:1 $\mathrm{H}_{2} \mathrm{O}: \mathrm{D}_{2} \mathrm{O}$ or $\mathrm{D}_{2} \mathrm{O}$ ratio. The Sørensen phosphate buffer at pH 5.8 was prepared with $92 \mathrm{~mL}$ of a solution $0.2 \mathrm{M} \mathrm{NaH}_{2} \mathrm{PO}_{4}$ and $8 \mathrm{~mL}$ of a $0.2 \mathrm{M}$ solution of $\mathrm{Na}_{2} \mathrm{HPO}_{4}$ using $\mathrm{D}_{2} \mathrm{O}$ as a solvent for both cases $[41,42]$. Solution $\mathrm{pH}$ values were verified using a $\mathrm{pH}$ electrode (Spintrode, Hamilton, Bonaduz, Switzerland) inside the NMR tube. Spectra were externally referenced to the singlet resonance of 4,4-dimethyl-4-silapentane-1-sulfonic acid (DSS) at $0 \mathrm{ppm}$. Spectra were recorded on a Bruker AVANCE-700 with a cryoprobe and an AVANCE-III 800. All the spectra were recorded using a sample with a concentration of $1.6 \mathrm{mM}$ at $300 \mathrm{~K}$. The experiments used were the, TOtal Correlation SpectroscopY (TOCSY), COrrelation SpectroscopY (COSY), Nuclear Overhauser Effect SpectroscopY (NOESY), Rotating frame Overhauser Effect SpectroscopY 
(ROESY), Heteronuclear Multiple Bond Correlation (HMBC), Heteronuclear Single Quantum Coherence $\left({ }^{15} \mathrm{~N}-\mathrm{HSQC}\right)$ Heteronuclear Multiple Quantum Coherence $\left({ }^{13} \mathrm{C}-\mathrm{HMQC}\right)$, and Double Quantum Filter-COrrelation SpectroscopY (DQF-COSY). Excitation sculpting sequence was used for water suppression. The mixing times of NOESY experiments were set to150-250 ms.

\subsection{Statistical Analysis}

The statistical analysis of the combined chemical shift deviations from random coil values was achieved by calculating RMSS (root of the mean of sum squares) of all available nuclei, namely $\mathrm{H}^{\mathrm{N}}$, $\mathrm{N}, \mathrm{H} \alpha, \mathrm{H} \beta, \mathrm{C} \alpha$ and $\mathrm{C} \beta$ involved for each amino acid except for glycines. The formula involves the use of correction factors calculated from the Biological Magnetic Resonance Data Bank (BMRB) statistics $[43,44]$ representing the average variances of the nuclei in relation with the gyromagnetic constant of each nuclei. The values considered for the analysis are the experimental chemical shift $(\delta \mathrm{Xexp})$ and the random coil shift $(\delta \mathrm{X} r c)$ while $n$ represents the number of terms used in this equation.

$$
R M S S=\sqrt{\frac{\left(1 *\left(\delta H_{e x p}-\delta H_{r c}\right)^{2}\right)+\left(0.185 *\left(\delta C_{e x p}-\delta C_{r c}\right)^{2}\right)+\left(0.156 *\left(\delta N_{e x p}-\delta N_{r c}\right)^{2}\right)}{n}}
$$

The random coil values used were obtained from Schwarzinger et al. [45]. The value of the RMSS gives a collective estimate of how far each amino acid is from the random coil, and it is not affected for the number of nuclei considered in each case.

The weighting factors used in the RMSS formula were obtained from the standard deviation of the respective values reported in the diamagnetic set of chemical shifts of the BMRB. These statistical deviations originated from comparable number of measurements.

\section{4. ${ }^{3} J_{H N-H \alpha}$ Couplings Analysis}

${ }^{3} J_{\mathrm{HN}-\mathrm{H} \alpha}$ couplings constants values were obtained from the DQF-COSY spectrum, by measuring the splitting of the antiphase peaks. The digital resolution of the spectra used was $1.8 \mathrm{~Hz} /$ point and the half height line width of the antiphase peaks ranged from $1.8-2.5 \mathrm{~Hz}$ which is less than two times the scalar couplings measured, limiting the experimental error to below 1\% [46]. This analysis uses an algorithm to build multiple, different copies of the same polypeptide chain by randomly sampling amino acid-specific backbone dihedral angle $\{\varphi / \psi\}$ potential wells. The peptide chain is constructed by using the selected $\{\varphi / \psi\}$ pairs to sequentially connect peptide planes. The calculation of the scalar ${ }^{3} J_{\mathrm{HN}-\mathrm{H} \alpha}$ considers the use of the following Karplus relationship to calculate the values for each conformer:

$$
{ }^{3} J_{H N-N \propto}(\varphi)=A \cos ^{2}\left(\varphi-60^{\circ}\right)+B \cos \left(\varphi-60^{\circ}\right)+C
$$

where $A=6.4, B=-1.4, C=1.9$ and $\theta=\varphi-60^{\circ}[47,48]$.

\section{Conclusions}

We have shown, despite the fluxional behavior of a small peptide a careful chemical shift analysis, using available data bank values, can identify structural features efficiently. The current NMR analysis of the Alzheimer's disease related peptides vaccines Ag85B $241-255-G P S L-T_{22}$ 29-237[pThr231/pSer235] 
demonstrated the potential presence of a $\beta$-turn comprising the linker sequence GPSL but no significant tertiary structure of the peptide vaccines. The conformation of the GPSL $\beta$-turn is not stable, but contributes to the overall structure and was therefore detectable. The GPSL sequence acted as a flexible linker joining the $\mathrm{B}$ cell and the $\mathrm{T}$ cell epitope, of the vaccine without transporting structural information to the other part. Contrary to this study, a local polyproline type II helix was previously reported for the phosphorylated Tau sequence [49] while the predicted structure of the tuberculosis T cell epitope is partially $\alpha$-helical [50]. However, our results agree with the observation that proline is an amino acid which promotes flexible structures [31]. The existence of $i, i+2$ NOEs in the region of the loop, is evidence of local organization and smaller conformational inhomogeneity than the rest of the molecule. These longer range NOEs can only be obtained if the amides involved retain their relative positions in the ms time scale which is not possible in a random coil environment. This tendency of preorganization is not observed in the rest of the molecule, and is in agreement with the presence of the loop. The efficacy of the vaccine to induce an immune response directed towards the double phosphorylation site, the specificity of the phosphorylation dependent antibodies and the role of the $\mathrm{T}$ cell epitope is subject of ongoing work and might result in a novel immunization strategy for the treatment of Alzheimer's disease. Preliminary data suggest that this first generation antigenic peptide has immunogenic properties despite the absence of significant structure. Future iteration of this peptide design will explore different structural elements and the combined results will allow us to make the correlations of structure and antigenic activity.

\section{Supplementary Materials}

${ }^{1} \mathrm{H}-700 \mathrm{MHz}$ spectrum of the phosphorylated peptide (Figure S1), TOtal Correlation SpectroscopY (TOCSY) spectrum (Figure S2). Chemical shift assignment of the AD-specific peptide vaccine Ag85B $241-255$-GPSL-Tau $229-237\left[\mathrm{pThr231/pSer235]} \mathrm{(Table} \mathrm{S1),}{ }^{3} J_{\mathrm{NH}-\mathrm{H} \alpha}\right.$ analysis from experimental data (Exp) and Flexible Meccano (FM) ensemble (Table S2).

Supplementary materials can be accessed at: http://www.mdpi.com/1420-3049/18/5/4929/s1.

\section{Acknowledgments}

K.R-G thanks to Consejo Nacional de Ciencia y Tecnología (CONACyT) for a scholarship. This work was supported by the German Research Foundation, SFB 610, Project A2 and the European Union and the Free State of Saxony (Grand number 080937179). The Portuguese National NMR Network (REDE/1517/RMN/2005) was supported by POCI 2010 and Fundação para a Ciência e a Tecnologia.

\section{References}

1. Mei, C.G.; Jahr, N.; Singer, D.; Berger, S. Hairpin conformation of an 11-mer peptide. Bioorg. Med. Chem. 2011, 19, 3497-3501.

2. Paine, G.H.; Scheraga, H.A. Prediction of the native conformation of a polypeptide by a statistical-mechanical procedure. I. Backbone structure of enkephalin. Biopolymers 1985, 24, 1391-1436. 
3. Blanco, F.J.; Jimenez, M.A.; Herranz, J.; Rico, M.; Santoro, J.; Nieto, J.L. NMR evidence of a short linear peptide that folds into a .beta.-hairpin in aqueous solution. J. Am. Chem. Soc. 1993, 115, 5887-5888.

4. Wright, P.E.; Dyson, H.J.; Lerner, R.A. Conformation of peptide fragments of proteins in aqueous solution: Implications for initiation of protein folding. Biochemistry 1988, 27, 7167-7175.

5. Mittag, T.; Forman-Kay, J.D. Atomic-level characterization of disordered protein ensembles. Curr. Opin. Struct. Biol. 2007, 17, 3-14.

6. Eliezer, D. Biophysical characterization of intrinsically disordered proteins. Curr. Opin. Struct. Biol. 2009, 19, 23-30.

7. Fesinmeyer, R.M.; Hudson, F.M.; Olsen, K.A.; White, G.W.N.; Euser, A.; Andersen, N.H. Chemical shifts provide fold populations nd register of beta hairpins and beta sheets. J. Biomol. NMR 2005, 33, 213-231.

8. Gross, K.-H.; Kalbitzer, H.R. Distribution of chemical shifts in $1 \mathrm{H}$ nuclear magnetic resonance spectra of proteins. J. Magn. Reson. 1988, 76, 87-99.

9. Spera, S.; Bax, A. Empirical correlation between protein backbone conformation and C.alpha. and C.beta. 13C nuclear magnetic resonance chemical shifts. J. Am. Chem. Soc. 1991, 113, 5490-5492.

10. Szilagyi, L.; Jardetzky, O. $\alpha$-Proton chemical shifts and secondary structure in proteins. J. Magn. Reson. 1989, 83, 441-449.

11. Meier, S.; Blackledge, M.; Grzesiek, S. Conformational distributions of unfolded polypeptides from novel NMR techniques. J. Chem. Phys. 2008, 128, 052204.

12. Wright, P.E.; Dyson, H.J. Linking Folding and Binding. Curr. Opin. Struct. Biol. 2009, 19, 31-38.

13. Jensen, M.R.; Markwick, P.; Griesinger, C.; Zweckstetter, M.; Meier, S.; Grzesiek, S.; Bernado, P.; Blackledge, M. Struct. Bonding 2009, 17, 1169-1185.

14. Hutchinson, E.G.; Thornton, J.M. A revised set of potentials for beta turn formation in proteins. Protein Sci. 1994, 3, 2207-2216.

15. Rose, G.D.; Glerasch, L.M.; Smith, J.A. Turns in Peptides and Proteins. Adv. Protein Chem. 1985, $37,1-109$.

16. Wilmot, C.M.; Thornton, J.M. Analysis and prediction of the different types of $\beta$-turn in proteins. J. Mol. Biol. 1988, 203, 221-232.

17. Kaumaya, P.T.; Foy, K.C.; Garrett, J.; Rawale, S.V.; Vicari, D.; Thurmond, J.M.; Lamb, T.; Mani, A.; Kane, Y.; Balint, C.R.; et al. Phase I active immunotherapy with combination of two chimeric, human epidermal growth factor receptor 2, B-cell epitopes fused to a promiscuous T-cell epitope in patients with metastatic and/or recurrent solid tumors. J. Clin. Oncol. 2009, 27, 5270-5277.

18. Menéndez-González, M.; Pérez-Piñera, P.; Martínez-Rivera, M.; Muñiz, A.L.; Vega, J.A. Immunotherapy for Alzheimer's disease: Rational basis in ongoing clinical trials. Curr. Pharm. Des. 2011, 17, 508-520.

19. Wolf, A.; Mozdzanowska, K.; Williams, K.L.; Singer, D.; Richter, M.; Hoffmann, R.; Caton, A.J.; Otvos, L.; Erikson, J. Vaccination with M2e-based multiple antigenic peptides: Characterization of the B cell response and protection efficacy in inbred and outbred mice. PLoS One 2011, 6, e28445.

20. Citron, M. Alzheimer's disease: Strategies for disease modification. Nat. Rev. Drug Discov. 2010, 9, 387-398. 
21. Braak, H.; Braak, E. Staging of Alzheimer's disease-related neurofibrillary changes. Neurobiol. Aging 1995, 16, 271-278.

22. Shah, S.; Federoff, H.J. Therapeutic potential of vaccines for Alzheimer's disease. Immunotherapy 2011, 3, 287-298.

23. Weiner, H.L.; Frenkel, D. Immunology and immunotherapy of Alzheimer's disease. Nat. Rev. Immunol. 2006, 6, 404-416.

24. Asuni, A.A.; Boutajangout, A.; Quartermain, D.; Sigurdsson, E.M. Immunotherapy Targeting Pathological Tau Conformers in a Tangle Mouse Model Reduces Brain Pathology with Associated Functional Improvements. J. Neurosci. 2007, 27, 9115-9129.

25. Sigurdsson, E.M.; Knudsen, E.; Asuni, A.; Fitzer-Attas, C.; Sage, D.; Quartermain, D.; Goni, F.; Frangione, B.; Wisniewski, T. An attenuated immune response is sufficient to enhance cognition in an Alzheimer's disease mouse model immunized with Amyloid- $\beta$ derivatives. J. Neurosci. 2004, 24, 6277-6282.

26. Dakappagari, N.K.; Douglas, D.B.; Triozzi, P.L.; Stevens, V.C.; Kaumaya, P.T.P. Prevention of Mammary Tumors with a Chimeric HER-2 B-cell Epitope Peptide Vaccine. Cancer Res. 2000, 60, 3782-3789.

27. Kaumaya, P.T.; Berndt, K.D.; Heidorn, D.B.; Trewhella, J.; Kezdy, F.J.; Goldberg, E. Synthesis and biophysical characterization of engineered topographic immunogenic determinants with alpha alpha topology. Biochemistry (Wash.) 1990, 29, 13-23.

28. Lairmore, M.D.; DiGeorge, A.M.; Conrad, S.F.; Trevino, A.V.; Lal, R.B.; Kaumaya, P.T. Human T-lymphotropic virus type 1 peptides in chimeric and multivalent constructs with promiscuous T-cell epitopes enhance immunogenicity and overcome genetic restriction. J. Virol. 1995, 69, 6077-6089.

29. Singer, D.; Lehmann, J.; Hanisch, K.; Hartig, W.; Hoffmann, R. Neighbored phosphorylation sites as PHF-tau specific markers in Alzheimer's disease. Biochem. Biophys. Res. Commun. 2006, 346, 819-828.

30. Vranken, W.F.; Boucher, W.; Stevens, T.J.; Fogh, R.H.; Pajon, A.; Llinas, M.; Ulrich, E.L.; Markley, J.L.; Ionides, J.; Laue, E.D. The CCPN data model for NMR spectroscopy: Development of a software pipeline. Proteins 2005, 59, 687-696.

31. Dunker, A.K.; Lawson, J.D.; Brown, C.J.; Williams, R.M.; Romero, P.; Oh, J.S.; Oldfield, C.J.; Campen, A.M.; Ratliff, C.M.; Hipps, K.W.; et al. Intrinsically disordered protein. J. Mol. Graph. Model. 2001, 19, 26-59.

32. Available online: http://deposit.bmrb.wisc.edu/author_view/BMRB/19112_hy_errejbgi.str (accessed on 19 April 2013)

33. Case, D.A.; Dyson, H.; Wright, P.E. Use of chemical shifts and coupling constants in nuclear magnetic resonance structural studies on peptides and proteins. Methods Enzymol. 1994, 239, 392-416.

34. Sharman, G.J.; Griffiths-Jones, S.R.; Jourdan, M.; Searle, M.S. Effects of the amino acid (phi, psi) propensities and secondary structure interactions in modulatin Halfa shift in peptide ans protein beta-sheet. J. Am. Chem. Soc 2001, 123, 12318-12324.

35. Wishart, D.S.; Sykes, B.D.; Richards, F.M. The chemical shift index: A fast and simple method for the assignment of protein secondary structure through NMR spectroscopy. Biochemistry 1992, $31,1647-1651$. 
36. Santiveri, C.; Rico, M.; Jiménez, M. $13 \mathrm{C} \alpha$ and $13 \mathrm{C} \beta$ chemical shifts as a tool to delineate $\beta$-hairpin structures in peptides. J. Biomol. NMR 2001, 19, 331-345.

37. Wishart, D.S.; Sykes, B.D. Chemical-Shifts As A Tool for Structure Determination. Nuclear Magnetic Resonance Pt. C 1994, 239, 363-392.

38. Bundi, A.; Wuthrich, K. H-1-Nmr parameters of the common amino-acid residues measured in aqueous-solutions of the linear tetrapeptides H-Gly-Gly-X-L-Ala-OH. Biopolymers (Biospectrosc.) 1979, 18, 285-297.

39. Modig, K.; Jurgensen, V.W.; Lindorff-Larsen, K.; Fieber, W.; Bohr, H.G.; Poulsen, F.M. Detection of initiation sites in protein folding of the four helix bundle ACBP by chemical shift analysis. FEBS Lett. 2007, 581, 4965-4971.

40. Karplus, M. Vicinal proton coupling in nuclear magnetic resonance. J. Am. Chem. Soc. 1963, 85, 2870-2871.

41. Gomori, G. Preparation of buffers for use in enzyme studies. Methods Enzymol. 1955, 1, 138-146.

42. Sørensen, S.P.L. Biochem. Z. 1909, 21, 131.

43. Biological Magnetic Resonance Data Bank. Available online: http://www.bmrb.wisc.edu/ ref_info/statsel.htm/ (accessed on 19 April 2013).

44. Ulrich, E.L.; Akutsu, H.; Doreleijers, J.F.; Harano, Y.; Ioannidis, Y.E.; Lin, J.; Livny, M.; Mading, S.; Maziuk, D.; Miller, Z.; et al. BioMagResBank. Nucleic Acids Res. 2008, 36, D402-D408.

45. Schwarzinger, S.; Kroon, G.J.A.; Foss, T.R.; Wright, P.E.; Dyson, H.J. Random coil chemical shifts in acidic $8 \mathrm{M}$ urea: Implementation of random coil shift data in NMRView. J. Biomol NMR 2000, 18, 43-48.

46. Cavanagh, J.; Fairbrother, W.J.; Palmer, A.G., III; Rance, M.; Skelton, N.J. Protein NMR Spectroscopy; Academic Press Inc.: San Diego, CA, USA, 2006.

47. Ozenne, V.; Bauer, F.; Salmon, L.; Huang, J.R.; Jensen, M.R.; Segard, S.; Bernado, P.; Charavay, C.; Backledge, M. Flexible-meccano: A tool for the generation of explicit ensemble descriptions of intrinsically disordered proteins and their associated experimental observables. Bioinformatics 2012, 28, 1463-1470.

48. Smith, L.J.; Bolin, K.A.; Schwalbe, H.; MacArthur, W.M.; Thornton, J.M.; Dobson, C.M. Analysis of main chain torsion angles in proteins: Prediction of NMR coupling constants for native and random coil conformations. J. Mol. Biol. 1996, 255, 494-506.

49. Bielska, A.A.; Zondlo, N.J. Hyperphosphorylation of tau induces local polyproline II helix. Biochemistry (Wash.) 2006, 45, 5527-5537.

50. D'Souza, S.; Rosseels, V.R.A.; Tanghe, A.; Denis, O.; Jurion, P.; Castiglione, N.; Vanonckelen, A.; Palfliet, K.; Huygen, K. Mapping of murine Th1 helper T-cell epitopes of mycolyl transferases Ag85A, Ag85B, and Ag85C from Mycobacterium tuberculosis. Infect. Immun. 2003, 71, 483-493.

Sample Availability: Not available.

(C) 2013 by the authors; licensee MDPI, Basel, Switzerland. This article is an open access article distributed under the terms and conditions of the Creative Commons Attribution license (http://creativecommons.org/licenses/by/3.0/). 